\title{
Tecnura
}

INVESTIGACIÓN

\section{Metodología para la formulación de proyectos basada en la definición del problema}

\author{
Problem-Definition-Based Methodology for Pre Formulation of Projects \\ Omar Iván Trejos Buriticá*
}

Fecha de recepción: 31 de marzo de 2014

Fecha de aceptación: 19 de enero de 2015

Como citar: Trejos Buriticá, O. I. (2015). Metodología para la formulación de proyectos basada en la definición del problema. Revista Tecnura, 19(45), 115-126. doi: 10.14483/udistrital.jour.tecnura.2015.3.a09

\section{RESUMEN}

El presente artículo plantea una metodología muy simple, sencilla y, hasta cierto punto, paramétrica para resolver el que se ha convertido en el problema más grande para los estudiantes de ingenierías que se encuentran en la recta final de su proceso de formación: la formulación del anteproyecto de su trabajo de grado y, a partir de allí, el planteamiento de un derrotero de actividades que permitan culminar exitosamente la propuesta. Durante mucho tiempo el autor ha impartido una gran cantidad de cursos, asesorías y acompañamientos de muchos estudiantes y ha encontrado una metodología simple para que la formulación de un anteproyecto deje de ser un terreno fangoso para convertirse en un camino casi paramétrico. Esa metodología es, precisamente, la que se plantea en este artículo.

Palabras clave: definición del problema, metodología, proyectos, trabajo de grado.

\begin{abstract}
This article shows a simple, parametric and effective methodology to solve the big problem of the Engineering Program's students when they are in the last semesters of his careers: the formulation of the preproject and, from there, the design of the activities' schedule to finish his studies successfully. For long, the author has been teacher, adviser and accompanist for a lot of students and he has found a simple methodology to formulate a preproject in an easy way. This is the target of this article.
\end{abstract}

Keywords: methodology, problem definition, projects, thesis.

\footnotetext{
* Ingeniero de sistemas, doctor en Ciencias de la Educación, Universidad Tecnológica de Pereira, Pereira, Colombia. Contacto: omartrejos@ utp.edu.co
} 


\section{INTRODUCCIÓN}

Una de las dificultades más grandes que tienen tanto estudiantes de pregrado como de posgrado, pero más notorio en los estudiantes de pregrado, es la formulación de su anteproyecto y, a partir de allí, la concreción de las actividades que permitan construir y culminar exitosamente su trabajo de grado (Hernández, 2008).

Según el Ministerio de Educación de Colombia en su informe sobre la retención y deserción universitaria de 2012, el 23\% de los estudiantes que ingresan a primer semestre en los programas de Ingeniería, Arquitectura, Urbanismo y afines desertan de su carrera; el $46 \%$ de la población restante lo hace durante el quinto semestre y el $55 \%$ deserta cuando se encuentran en décimo semestre. Si bien pudiera hacerse un análisis detallado de los factores que influyen en estas decisiones, este artículo se ocupará de las razones por las cuales los estudiantes de décimo semestre, quienes ya están próximos a terminar su proceso de formación, desertan y no se gradúan (Agencia Presidencial de Cooperación Internacional, 2012).

Para precisar las cifras debe entenderse que de cada 100 estudiantes que ingresan a las carreras de Ingeniería y afines, 23 de ellos aproximadamente desertan. De los 77 restantes, el 46\% (o sea, casi la mitad) deserta cuando están cursando quinto semestre, por lo cual quedan 38 estudiantes aproximadamente de la cohorte original. De estos 38 estudiantes, el 55\% deserta cuando va en décimo semestre, es decir que culminan exitosamente su carrera tan solo 17 estudiantes aproximadamente. Esto nos induce a pensar que, en cifras aproximadas, de cada 100 estudiantes que ingresan a un programa de Ingeniería y afines, tan solo 17 lo culminan exitosamente. Debe anotarse que, en décimo semestre, el porcentaje de deserción de los programas de Ingeniería y afines es el más alto si se compara con los porcentajes equivalentes (esto es, deserción porcentual cuando los estudiantes están en décimo semestre) de otras áreas: Bellas Artes (52\%); Administración,
Economía y afines (50\%); Agronomía, Veterinaria y afines (50\%); Ciencias de la Educación (47\%); Ciencias Básicas (46\%); Ciencias Sociales (45\%) y Ciencias de la Salud (38\%) (Agencia Presidencial de Cooperación Internacional, 2012).

Aunque el Ministerio de Educación Nacional ha puesto en funcionamiento diferentes estrategias, tales como el Sistema SPADIES (Sistema para la Prevención de la Deserción en la Educación Superior) para disminuir la deserción en los últimos semestres, la tendencia se mantiene. Según el sitio web oficial del Ministerio de Educación (2014), la deserción entre el décimo semestre cursado y el décimo quinto semestre cursado en programas de Ingeniería, Arquitectura, Urbanismo y afines tiene una tendencia a superar el $50 \%$ de los estudiantes. Las razones que generan la deserción en unos semestres tan avanzados como el décimo cursado, y de allí en adelante, podrían ser muchas, sin embargo la experiencia institucional de acompañamiento del autor en una gran cantidad de universidades le permite advertir que incide significativamente el hecho de que los estudiantes no terminan su programa de formación por el reto que constituyen el hallazgo de un tema, la formulación de su anteproyecto, el acompañamiento de un buen docente, la poca formación en comunicación escrita y la falta de una metodología que hile todos estos elementos para que lo haga simple y sencillo de digerir y de ejecutar (Lerma, 2005).

El propósito de este artículo radica en la formulación de una metodología que atienda esta ingente necesidad y que ponga a disposición de los estudiantes y de los docentes una metodología que permita definir un problema, formular un anteproyecto y desarrollar el trabajo de grado por los caminos paramétricos que, en muchos casos, la Ingeniería pareciera exigir. Podría decirse que este artículo se justifica en virtud de la gran cantidad de esfuerzos que el Ministerio de Educación y las instituciones, en los campos académico e investigativo, están haciendo para propiciar esos caminos que propendan por simplificar la formulación del anteproyecto y el desarrollo del trabajo de grado, 
sin arriesgar la calidad académica. El aspecto innovador de esta propuesta radica en que se plantean unos criterios que facilitan tanto el hallazgo de un problema a partir de las preferencias de los estudiantes, como la formulación del anteproyecto y el desarrollo del documento final, llámese trabajo de grado, tesis o cualquier otro documento de alto nivel. El artículo se concentra en proponer una metodología para hallar efectivamente un problema o tema que sirva como insumo para formular el anteproyecto y en una metodología que simplifique y facilite dicha formulación partiendo de la definición del problema.

Para plantear el contenido de este artículo se ha acudido a la experiencia, por parte del autor, de más de veinte años de acompañamiento académico a estudiantes de todos los niveles de formación universitaria, a los factores comunes que han ido encontrando a lo largo de ese tiempo, tanto en la concepción de las diferentes ideas como en las políticas institucionales de las universidades del Eje Cafetero y a la búsqueda permanente de mecanismos que simplifiquen la formulación de anteproyectos, teniendo en cuenta los problemas que implican las cifras porcentuales de la deserción y la gran debilidad que se tiene en materia de comunicación escrita en las universidades.

Ante la pregunta formulada a manera de hipótesis para este artículo ¿es posible encontrar una metodología que simplifique el hallazgo de un problema por investigar y la formulación de un anteproyecto que cristalice lo propuesto? La respuesta es completamente afirmativa y ese es el propósito de este artículo. Este texto es uno de los productos de la tesis doctoral "Aprendizaje en Ingeniería: un problema de incomunicación", con la cual el autor obtuvo su título de doctor en Ciencias de la Educación en RUDEColombia Cade UTP, que fue registrado como proyecto de investigación ante la Vicerrectoría de Investigaciones de la Universidad Tecnológica de Pereira. Este artículo plantea la metodología propuesta bajo el estándar IMRYD (Introducción, Metodología, Resultados y Discusión) tanto en la selección de un tema por investigar como en la estructura de formulación de un anteproyecto, posteriormente presenta algunas estadísticas de aplicación de esta metodología, hace alguna discusión al respecto de los resultados obtenidos y finalmente plantea unas conclusiones que resumen el contenido del artículo.

\section{METODOLOGÍA}

\section{Metodología para seleccionar un tema}

El primer problema con el cual se enfrenta el estudiante radica en encontrar un tema para abordar de cara a la construcción de su documento de trabajo de grado. ¿Qué hacer para graduarme? es la pregunta que, con frecuencia, se hace y que, también con frecuencia, no siempre encuentra una respuesta.

En este sentido debe aclararse que la mayoría de universidades han adoptado diferentes líneas como trabajo final en los programas de pregrado de las áreas de ingenierías, que incluyen el desarrollo de monografías, la realización de proyecto que implique aplicación de conocimiento, el desarrollo de un proyecto de investigación, la creación de empresas, la práctica universitaria e, incluso, la realización del primer semestre de una especialización o una maestría ofrecida por la misma institución (Salinas, 2005). Todas estas son modalidades validadas internacionalmente que muchas universidades en el mundo han adoptado y que también se han incorporado en nuestro país, algunas con más intensidad que otras. Este artículo se ocupa de la formulación del documento final de las opciones aplicación de conocimiento, desarroIlo de un proyecto de investigación y monografía, es decir, todo lo dicho aquí es aplicable a estas tres opciones de grado. La base teórica y lógica que subyace a la propuesta también puede ser extrapolada, ajustada y aplicada a las otras formas de grado que proveen las instituciones (Cortés \& Iglesias, 2004).

El primer paso para construir un anteproyecto radica en seleccionar un tema para investigar o 
sobre el cual se quiere desarrollar una monografía o una aplicación de conocimiento (más conocida como trabajo de grado o tesis para los niveles de maestría y doctorado). La metodología se remite a que el estudiante realice de una manera concienzuda y franca consigo mismo los siguientes pasos:

- Listar las tres asignaturas o tres áreas que más le gustaron durante su ciclo de formación.

- Seleccionar sus palabras clave (posiblemente estas correspondan al nombre de las mismas asignaturas).

- Traducirlas, preferiblemente, al inglés, aunque no de manera exclusiva, esto es, puede acudirse a otro idioma.

- Utilizar tanto las palabras en español como su traducción como filtro de búsqueda en bases de datos especializadas.

- Comenzar a revisar el nombre de artículos que incluyan las tres palabras.

- Leer detenidamente el resumen (Ilamado también "Abstract") para tener una aproximación más clara a lo que contiene el artículo.

- De aquellos artículos que se considere que coinciden con nuestras expectativas, leer la Introducción (normalmente el numeral 1 del contenido del artículo).

- Seleccionar dos o tres temas que llamen la atención.

Vale la pena tener en cuenta que una vez se ejecute de manera secuencial y precisa esta metodología, se puede garantizar que el estudiante (preferiblemente aquel que no sabía qué hacer como trabajo de grado) habrá encontrado un tema que cumple con las características como para que su desarrollo le permita concluir su proceso de formación profesional en pregrado de ingenierías (McConell, 2005).

\section{Cuatro reflexiones}

El Dr. Guillermo Hoyos, ícono de la investigación en Colombia y fallecido a principios del año
2014, investigador y filósofo que compartió escenario con los pensadores más prominentes de la última década a nivel mundial, siempre hacía tres recomendaciones que vale la pena reproducir para beneficio de todos aquellos que accedan a este artículo. Sus palabras, producto de una muy profunda experiencia en el campo de la investigación y en los procesos de acompañamiento de estudiantes de pregrado, especialización, maestrías y doctorados, permitían tener en cuenta lo que él mismo llamaba "las claves para construir un trabajo de grado de manera eficiente":

- Jamás comprometerse a desarrollar un trabajo de grado o una tesis sobre un tema que no se conozca.

- Jamás comprometerse a desarrollar un trabajo de grado o una tesis sobre un tema que no sea del agrado total para el estudiante.

- Jamás comprometerse a desarrollar un trabajo de grado o una tesis con alguien con quien no quiera trabajar (eso incluye tanto a compañeros como al director del trabajo de grado).

Sencillas pero efectivas las recomendaciones del Dr. Hoyos y, con alguna experiencia acumulada, el autor de este artículo puede asegurar que efectivamente son las "claves" para construir el trabajo de grado o la tesis que se quiera desarrollar.

La segunda reflexión va de la mano de la experiencia acumulada por el autor de este artículo. Lo más importante de una propuesta o de un anteproyecto no es lo que se quiere hacer, sino la coherencia de la metodología como se quiere hacer, es decir, el sentido lógico que tienen los pasos con los cuales se quiere abordar y resolver un problema determinado. El QUÉ pasa a un segundo plano cuando el CÓMO es lógico y completamente realizable. La historia de la ciencia da fe de esto último, pero se omitirá por ser un tema fuera de los linderos del contenido de este artículo.

La tercera reflexión acude a la fuente de consulta excelsa para conocer investigaciones 
recientes al respecto de un tema determinado y para tener la posibilidad de contactar investigadores con quienes se pueda interactuar alrededor de un determinado tema: las bases de datos especializadas. Estas bases de datos se han constituido en la base para acceder al soporte documental de cualquier investigación, dado que cuentan con un sistema de arbitraje que garantiza que el contenido de los artículos publicados tiene el nivel académico necesario y la actualidad pertinente como para tenerlos como referentes bibliográficos que oficien como punto de partida del tema seleccionado, además de formar parte del respectivo estado del arte. Algunas de estas bases de datos especializadas, tal vez las más populares, son IEEExplore, ACM Library, ProQuest y Science Direct.

La cuarta reflexión hace referencia a la distribución del tiempo, a la forma como se utilice como recurso en la construcción de un trabajo de grado y a la manera como el estudiante puede llegar a ser altamente productivo en la construcción de su documento de trabajo de grado. Una estrategia es identificar en qué momento del día es más productivo cada estudiante y destinar una hora para tal fin, de manera que en esa hora y solo en ella el tiempo sea invertido en la confección de la tesis o del trabajo de grado.

Si usted destina una hora diaria (de alta productividad) al trabajo de grado, tendrá en la semana 7 horas de alta productividad, en el mes tendrá aproximadamente 28 horas de alta productividad y en seis meses tendrá alrededor de 160 horas de alta productividad. Esto representa mucho más de lo que usted haría dedicando todo un día continuo en su tesis o trabajo de grado.

Estudios recientes han demostrado que una hora de alta productividad equivale a seis horas de productividad media o a diez horas de baja productividad. La conversión habla por sí sola. Lo único que debe hacer el estudiante es darse a la tarea de identificarla y dedicarla a su trabajo de grado.

\section{Metodología para formular un anteproyecto}

Lo primero que debe tenerse en cuenta es que todo trabajo de grado o toda tesis de maestría o doctorado surgen de una idea, luego la primera acción por realizar consiste en escribir la idea con la extensión y la amplitud que considere conveniente, con el nivel de detalle que se elija y con los términos, expresiones, adjetivos y sustantivos que a bien tenga escribir.

Mejor dicho, escribir la idea tal como el estudiante lo considere, sin pensar en que está escribiendo la idea de un trabajo de grado o de una tesis. Escribirla como "venga en gana", sin condiciones. Es de anotar que la idea debe acudir a la descripción informal de un problema que se quiere abordar y cuya solución es la que esperamos constituya el corpus del trabajo de grado o la tesis.

Una vez escrita la idea, sin condiciones, sin reglas y sin cortapisas, se procede a volverla a escribir, pero esta vez con los siguientes parámetros: escribirla de nuevo en solo dos páginas, con letra arial de 12 puntos, interlineado 1,5, márgenes estándar (los márgenes por defecto) e intentando no superar seis párrafos en la exposición.

Si se nota que es imposible reducir la idea a este formato, entonces ese será el primer indicio de que la idea no está suficientemente clara y entonces deberá investigarse un poco más al respecto. Si por el contrario es posible reescribir la idea con las condiciones expuestas, entonces eso significa que un destello de claridad emerge indicando que la idea tiene un nivel de claridad suficiente como para avanzar al siguiente paso.

El próximo paso consiste en volver a escribir la idea, pero con el siguiente esquema:

- Antecedentes. Identifique cuáles son los antecedentes del problema que usted quiere abordar, es decir, de dónde proviene el problema. En esta parte la información consultada debe ser verificable y basarse en fuentes confiables. Toda la información estadística que le dé soporte a los antecedentes será bienvenida. 
- Efectos. Explique con la mayor claridad posible los efectos de dichos antecedentes, es decir, qué dificultades se han generado porque no se ha haIlado una solución a dichos antecedentes. Aquí se plantea el problema en términos generales o el macroproblema que se quiere abordar, así como su contexto, es decir, el entorno sobre el cual el problema tiene efecto.

- Problema. En esta parte se plantea el problema que se quiere resolver y que no necesariamente debe coincidir con el que se quiere abordar. Esto es, el problema que se quiere abordar es el planteamiento general que implica una serie de situaciones imbuidas en el problema como tal; el problema a resolver puede ser una o dos de esas situaciones. Si el problema que se quiere abordar hiciera referencia a las dificultades de tráfico de una gran ciudad, un problema por resolver podría ser la sincronización de los semáforos que, a todas luces, es solo un pequeño subproblema del macroproblema planteado.

- Solución. En términos generales, y sin acudir a detalles minúsculos, se plantea la solución que se quiere implementar y que constituye el corpus del trabajo de grado o de la tesis, así como su aporte.

El siguiente paso consiste en escribir cuatro párrafos distribuidos en dos páginas tamaño carta: en la primera página se escriben los antecedentes y los efectos de dichos antecedentes, en la segunda se escribe el problema y la solución en términos generales. Se debe anotar que el espacio que ofrecen dos páginas, además de las condiciones de letra mencionadas, no son suficiente para hacer una exposición de manera amplia y detallada, sin embargo es necesario tener en cuenta que si un estudiante es capaz de escribir un problema en estos términos, en esas condiciones y en ese espacio, será la confirmación de que, definitivamente, tiene muy claro lo que quiere hacer.

Lo contrario también aplica, es decir, si un estudiante no es capaz condensar su problema en estas condiciones, deberá revisar un poco mejor lo que quiere hacer porque significa que no lo tiene muy claro. Debe acotarse que un párrafo no debe exceder las 12 líneas. A este esquema se le Ilamará Definición del Problema.

Una vez escrita la Definición del Problema en los términos que se explican, se procede a completar los cuatro pilares de un anteproyecto (la definición del problema es uno de ellos). Los otros tres pilares son: el Objetivo General, el Nombre del proyecto y la Justificación. El Objetivo General sale del tercer párrafo de la Definición del Problema, es decir, del párrafo en donde se formula el problema que se quiere resolver (no el problema que se quiere abordar, pues este sería el macroproblema). El Nombre del trabajo de grado o de la tesis sale del Objetivo General y podría decirse que la diferencia es puramente semántica: mientras que el Objetivo General comienza con un verbo, el Nombre de la tesis comienza con el sustantivo equivalente a ese verbo.

Si el Objetivo General del anteproyecto está descrito como "Formular un modelo matemático para describir el movimiento aleatorio de una pluma llevada por el viento", el Nombre será "Formulación de un modelo matemático para describir el movimiento aleatorio de una pluma llevada por el viento". Nótese que la relación entre el Objetivo General y el Nombre es absolutamente íntima y así debe ser. En caso de que se encuentren discrepancias o diferencias entre el Objetivo General y el Nombre del anteproyecto, será muy recomendable que se revise la definición del problema porque esas diferencias significarán que la Definición del Problema no está suficientemente clara.

Es de anotar que del Objetivo General se obtiene el filtro de búsqueda que se ha de usar en las bases de datos. Para ello basta con hacerle un análisis de contenido a dicho objetivo general. Si dice "Formular un modelo matemático para describir el movimiento aleatorio de una pluma llevada por el viento", entonces pueden destacarse las siguientes palabras: (modelo matemático)+(movimiento aleatorio $)+($ viento $)$. Si las traducimos, entonces el 
equivalente que obtendríamos sería: (mathematical model)+(random movement)+(wind) y con esto se obtiene el filtro de búsqueda tanto en español como en inglés, con el ánimo de que al buscar en las bases de datos especializadas se obtengan los artículos más cercanos al tema propuesto.

La Justificación, que es el cuarto pilar fundamental para la formulación, revisión y evaluación de un anteproyecto, consiste en exponer las razones por las cuales un estudiante de un programa de Ingeniería debe dedicar su tiempo y sus conocimientos a estudiar la solución a un problema determinado. Entre estas razones se destacan las siguientes:

- Razones tecnológicas. Con el desarrollo del proyecto, ¿qué nuevo uso se está proponiendo dar a determinadas tecnologías que aún no se haya dado?

- Razones humanísticas. Con el desarrollo del proyecto, ¿qué condiciones se podrían mejorar en la existencia, convivencia y permanencia del ser humano como ente social?

- Razones económicas. Con el desarrollo del proyecto, ¿qué razones de inversión pueden optimizarse, mejorarse, evaluarse, simplificarse o multiplicarse positivamente que todavía no se hayan tenido en cuenta?

- Razones ambientales. Con el desarrollo del proyecto, ¿qué condiciones ecológicas y del medio ambiente se pueden mejorar para el bienestar de la humanidad que aún no se hayan aprovechado?

- Razones académicas. Con el desarrollo del proyecto, ¿qué nuevos conocimientos se pueden difundir, "vulgarizar", acceder o divulgar a los cuales hasta ahora no se haya accedido?

- Razones sociales. ¿Cómo se beneficia la sociedad, en su conjunto, con el desarrollo e investigación sobre el tema?

Se debe anotar que otras razones pueden emerger dependiendo de la temática o del problema que se quiere abordar y del problema puntual que se quiera resolver, pero, en su gran mayoría, las propuestas de trabajo de grado de Ingeniería acuden a algunas de estas líneas como razones para su justificación.

También debe tenerse en cuenta que no es necesario que todas las razones aparezcan al momento de justificar un tema de investigación, de trabajo de grado o de tesis. Es muy importante saber que, cualquiera que sea la razón que se exponga como justificación (sea una o varias), deberá ir sustentada con estadísticas, referencias y fuentes confiables que refuercen lo que se escriba en el documento.

Teniendo definidos los cuatro pilares del proyecto en su fase de formulación previa (anteproyecto), se procede a realizar una derivación conceptual de cada uno de ellos, de la siguiente forma:

- Del Nombre se derivan los siguientes ítems:

- Marco Teórico, que corresponde al conjunto de conocimientos que le dan piso científico al tema y que subyacen al problema puntual que se quiere solucionar.

- Marco Conceptual, que corresponde a los conceptos clave que constituyen la base para el Marco Teórico; son esos conceptos los que permiten que dicho Marco Teórico exista.

- Marco Legal, que está formado por todas aquellas leyes, normas o disposiciones de orden legal (nacional e internacional) que rigen, regulan, determinan, verifican y retroalimentan la posible solución que se quiere implementar, el problema puntual que se quiere resolver y el problema general que se quiere abordar.

- Estado del Arte, que consiste en una breve relación de los estudios, investigaciones, tesis y documentos que se hayan publicado recientemente en relación con el tema que se quiere tratar, con la solución que se quiere implementar, con el problema puntual que 
se quiere resolver y con el problema general que se quiere abordar. En la confección del Estado del Arte son determinantes las bases de datos electrónicas.

- Del Objetivo General se derivan los siguientes ítems:

- Objetivos Específicos. Corresponde a los pasos medianamente generales que deben realizarse para cumplir con el objetivo general; no son actividades puntuales, pues deben escribirse con cierto nivel de generalidad sin que desplacen al objetivo general en su concepción.

- Recursos Necesarios. Consiste en la descripción de los recursos financieros, técnicos y humanos que se necesitan para cumplir con cada uno de los objetivos planteados.

- Cronograma. Consiste en la descripción temporal, es decir, cuánto tiempo tomará realizar cada uno de los objetivos específicos si se cuenta con estos.

- Referencias Bibliográficas. Corresponde a la referenciación (con normas) de los documentos, artículos, investigaciones, tesis y libros que le dan soporte a la base teórica investigativa que subyace tanto a la solución como al problema puntual por resolver, así como al problema general por abordar. Normalmente en Colombia se acude a las normas de referenciación APA o Icontec.

- Esquema Temático. Es la propuesta de Tabla de Contenido de la tesis o el trabajo de grado que se deriva de los objetivos, contando con los recursos apropiados en el tiempo indicado y tomando como base las Referencias Bibliográficas citadas.

- De la Justificación se derivan los siguientes ítems:

Tabla 1. Estimación en páginas de cada ítem

\begin{tabular}{lc}
\hline \multicolumn{1}{c}{ Ítem } & Número máximo de páginas. \\
\hline Definición del Problema & 2,0 \\
\hline Nombre & 0,5 \\
\hline Objetivo General & 0,5 \\
\hline Justificación & 2,0 \\
\hline Marco Teórico & 4,0 \\
\hline Marco Conceptual & 2,0 \\
\hline Marco Legal & 4,0 \\
\hline Estado del Arte & 4,0 \\
\hline Objetivos Específicos & 0,5 \\
\hline Recursos* & 1,0 \\
\hline Cronograma & 1,0 \\
\hline Referencias Bibliográficas & 1,0 \\
\hline Esquema Temático & 1,0 \\
\hline Diseño Metodológico & 0,5 \\
\hline Hipótesis & 0,5 \\
\hline Variables & 0,5 \\
\hline Instrumentos** & 25,0 \\
\hline Total páginas &
\end{tabular}

(*) Cuando los recursos se presentan en formato prediseñado de presupuesto, el estimativo de páginas puede cambiar.

(**) No existe un estimativo de páginas en los instrumentos, ya que estos pueden llegar a ser varios dependiendo de la investigación o tema propuesto.

Fuente: elaboración propia. 
- Diseño Metodológico. Consiste en la forma metodológica como se va a resolver el problema puntual, es decir, en la manera como se va a aplicar un método que se base en procesos científicos para darle toda la confiabilidad a la solución. Es el conjunto de pasos que se van a realizar para implementar la solución. En esta parte es importante tener en cuenta el enfoque cuantitativo, el enfoque cualitativo y el enfoque cualito-cuantitativo.

- Hipótesis. Corresponde al planteamiento del objetivo general en forma de pregunta. La hipótesis es la apuesta que se hace en investigación cuando un estudiante se propone resolver un problema puntual del cual considera que tiene la solución. Siempre será una incógnita que se espera resolver. No resolverla no invalida el tema como tal, pues cuando una hipótesis no se comprueba puede ser que no se ha definido bien el problema o que el camino metodológico que se escogió no fue el apropiado para resolverlo. En el segundo caso, se habrá encontrado un camino por donde el problema no se puede solucionar y eso es tan valioso como si la hipótesis se hubiera comprobado exitosamente.

- Variables. Factores que inciden de manera directa o indirectamente para que la solución pueda ser implementada y el problema puntual pueda ser resuelto

- Instrumentos. Son las encuestas, entrevistas, formatos, preguntas o formularios que se necesitan para recolectar información importante y pertinente en relación con la solución que se plantea, con el problema puntual por resolver o con el problema general por abordar. La tabla 1 proporciona una estimación máxima en páginas por ítem.

Cuando se plantea un valor como 0,5 se refiere al espacio aproximado que ocupa media página tamaño carta (estos son solo estimativos, pues es posible que algunos ítems como la descripción detallada de recursos financieros — también Ilamada Presupuesto-, los Instrumentos y el Marco Teórico puedan llegar a ser bastante amplios en comparación con dichos estimativos). Las normas de presentación de anteproyectos establecen que un buen estimativo en extensión es de 25 páginas (o una cantidad alrededor de ese valor, entre 20 y 30 páginas). Este estimativo se hace teniendo en cuenta letra arial entre 10 y 12 puntos, interlineado a 1.5 y márgenes estándar. Cualquiera de estos valores estimados que sea difícil de cumplir puede ser sinónimo de que no se tiene completamente claro lo que significa el ítem como tal y, al tiempo, es muy posible que sea sinónimo de que la Definición del Problema (o sea, la concreción del problema puntual por resolver) puede estar difusa, y por tanto se hace difícil precisar algunos de los otros ítems. Sin asegurar que sea esa la única razón, debe tenerse en cuenta al momento de formular su anteproyecto. Razones como el desconocimiento del tema, la falta de acceso a fuentes de información o a canales que posibiliten esas fuentes y la falta de acceso a tecnología también pueden ser factores que se develen al momento de formular un anteproyecto y que verifican que estos valores estimados no se cumplen fácilmente. Así como se ha establecido una relación de dependencia direccional entre la Definición del Problema y las otras tres bases de un anteproyecto (Objetivo General, Nombre y Justificación), y también se ha establecido una relación de subordinación de los otros ítems frente a estos pilares de la formulación, debe entenderse que la falta de concreción de algún ítem podría develar falta de claridad en el ítem del cual procede o del cual, teóricamente, debe derivarse.

\section{Esquema general}

Con el planteamiento anterior, el esquema equivalente se muestra en la figura 1. 


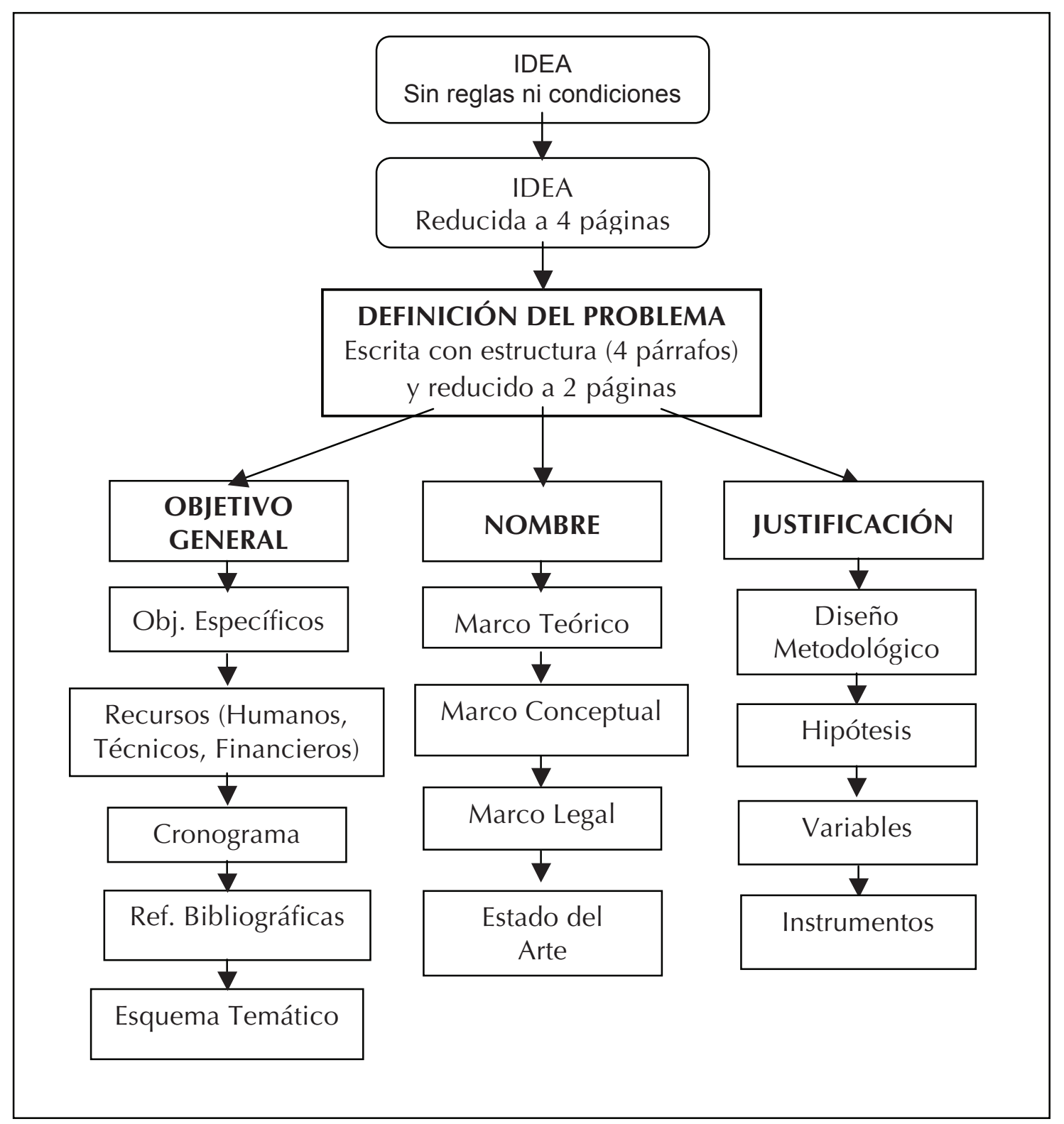

Figura 1. Esquema metodológico.

Fuente: elaboración propia.

\section{RESULTADOS}

Esta metodología para la formulación de anteproyectos de pregrado y posgrado ha sido utilizada en la Universidad Tecnológica de Pereira en el pregrado de Ingeniería de Sistemas y Computación, en la Especialización en Redes de Datos y en la
Maestría en Ingeniería de Sistemas, considerando las diferencias propias de cada nivel de formación. Ha sido utilizada con los estudiantes y los resultados han sido bastante favorables, dado que en tiempos recientes la formulación del anteproyecto le tomaba a más de la mitad de los estudiantes un tiempo superior a un semestre. La dificultad no se 
presentaba en lo que los estudiantes debían escribir, sino en la ausencia de una metodología clara que les permitiera tener un camino concreto que facilitara dicha escritura. Efectivamente, tal como se muestra en la tabla 2, los resultados han cambiado sustancialmente.

\section{DISCUSIÓN}

Tal como lo muestra la tabla 2, la relación porcentual entre los datos con y sin metodología es sustancialmente diferente. Mientras que en la maestría un total de 32 estudiantes, a lo largo de cuatro semestres, tenían la posibilidad de presentar su anteproyecto dentro de los límites de tiempo establecidos, solamente 10 de ellos lo lograron dentro del mismo semestre. La relación entre los estudiantes sin metodología que presentaron su anteproyecto frente al total de estudiantes que tenían la posibilidad de hacerlo no supera el 30\%.

Por su parte, de los 34 estudiantes que durante los cuatro semestres pudieron presentar su anteproyecto de maestría y fueron beneficiados con la metodología, 29 presentaron su anteproyecto dentro del mismo semestre, lo cual representa una efectividad del $85 \%$. Aunque debe admitirse que otros factores pueden influir en la formulación y presentación de los anteproyectos, no se puede negar (según lo dicho por los mismos estudiantes) que la utilización de una metodología tan concreta como la que se presenta en este artículo facilita mucho la formulación y trámite del anteproyecto en mención. Es de anotar que en esta tabla no se diferencian los estudiantes que definitivamente decidieron no presentar su anteproyecto; solo se acude a la estadística de quienes tenían posibilidad de presentarlo y lo hicieron dentro del marco metodológico aquí expuesto y por fuera de él.

En el caso de la Especialización en Redes de Datos, el resultado es muy similar. De 25 estudiantes que tenían posibilidad de presentar el anteproyecto, sin contar con la metodología presentada en este artículo, solo 10 lo presentaron (efectividad del $40 \%$ ). En cambio, de 25 estudiantes que fueron preparados con la metodología, 24 lo presentaron en un plazo máximo de un semestre (efectividad del 96\%). En el programa de pregrado en Ingeniería de Sistemas, la diferencia fue todavía más notoria. De 128 estudiantes que podían presentar su anteproyecto, solo 46 lo hicieron en los grupos que no tuvieron preparación con la metodología (efectividad del 36\%). Aquellos que fueron preparados con la metodología presentaron su anteproyecto en un solo semestre 95 de 97 estudiantes, lo

Tabla 2. Resultados comparativos

\begin{tabular}{llllllllllllll}
\hline & \multicolumn{4}{l}{ Pendientes por anteproyecto* } & \multicolumn{4}{l}{ Presentaron anteproyecto en un semestre } \\
\hline & \multicolumn{3}{l}{ Sin metodología } & \multicolumn{3}{l}{ Con metodología } & \multicolumn{3}{l}{ Sin metodología } & \multicolumn{3}{c}{ Con metodología } \\
\hline Sem & MISC & ERD & ISC & MISC & ERD & ISC & MISC & ERD & ISC & MISC & ERD & ISC \\
\hline I 2012 & 9 & 5 & 42 & 12 & 7 & 25 & 4 & 3 & 11 & 11 & 6 & 24 \\
\hline II 2012 & 8 & 6 & 30 & 8 & 7 & 29 & 2 & 2 & 14 & 7 & 6 & 29 \\
\hline I 2013 & 8 & 5 & 25 & 7 & 7 & 22 & 2 & 2 & 11 & 5 & 5 & 22 \\
\hline II 2013 & 7 & 9 & 31 & 7 & 4 & 21 & 2 & 3 & 10 & 6 & 4 & 20 \\
\hline
\end{tabular}

Fuente: elaboración propia.

MISC = Maestría en Ingeniería de Sistemas y Computación.

ERD = Especialización en Redes de Datos.

ISC = Ingeniería de Sistemas y Computación.

* Estudiantes que se encontraban en últimos semestres $\left(9^{\circ}\right.$ y $\left.10^{\circ}\right)$ y aún no habían presentado el anteproyecto. 
cual deja una estadística de efectividad del 97\%. Debe anotarse que estas cifras han ido mejorando a medida que los estudiantes se han ido capacitando en la metodología, ya que se ha notado el beneficio de formular un anteproyecto a partir de tener absolutamente clara la Definición del Problema.

\section{CONCLUSIONES}

Es posible generar alta motivación en la fase final de un proceso de formación a partir de la capacitación de los estudiantes en una metodología que simplifique y aclare la formulación de un anteproyecto.

- Es posible que un anteproyecto se formule apropiadamente en tiempos menores a los supuestamente estimados, esto es, en tiempos menores a un semestre. Así mismo, que para que un anteproyecto pueda ser formulado y presentado en tiempo récord, todo lo que se necesita es contar con una metodología suficientemente clara y concreta que, a partir de parámetros conceptuales claros, le posibilite al estudiante tanto la redacción como la interrelación entre sus componentes.

- Una necesidad ingente en las universidades colombianas es preparar a los estudiantes en redactar, formular, proponer $y$, en general, escribir de manera que puedan comunicarse por escrito tan bien como lo pueden hacer verbalmente.

- Conocer y adoptar una metodología para formular anteproyectos es el primer paso firme para que el desarrollo del trabajo de grado o de la tesis tenga una base clara y concreta: la definición del problema, y que cualquier formulación de un trabajo de grado o de una tesis puede desarroIlarse a partir de un principio muy sencillo: tener clara la definición del problema.

\section{FINANCIAMIENTO}

Universidad Tecnológica de Pereira

\section{REFERENCIAS}

Agencia Presidencial de Cooperación Internacional. (2012). Manual de formulación de proyectos. Bogotá, Colombia: Presidencia de la República.

Briones, G. (1996). Metodología de la investigación cuantitativa en ciencias sociales. Bogotá, Colombia: Instituto Colombiano para el Fomento de la Educación Superior.

Cortés, M. \& Iglesias, M. (2004). Generalidades sobre metodología de la investigación. Ciudad del Carmen, Campeche. México: Universidad Autónoma del Carmen.

Hernández, R. (2008). Metodología de la investigación. Ciudad de México: McGraw Hill Interamericana.

Lerma, H. (2009). Metodología de la investigación. Bogotá, Colombia: Ecoe Ediciones.

McConell, S. (2005). Desarrollo de proyectos informáticos. NY, USA: McGraw Hill Companies.

Ministerio de Educación Nacional. (2014). www.mineducacion.gov.co.

Sabino, C. (1992). El proceso de investigación. Bogotá, Colombia: Editorial Panapo. Salinas, P. (2005). Metodología de la investigación científica. Mérida, Venezuela: Universidad de los Andes.

\section{(C) $(1) \Theta$}

